\title{
Hepatocellular carcinoma: asphyxiate and then cook
}

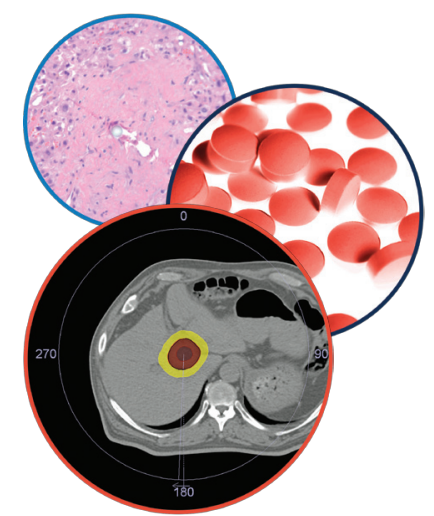

Hepatic Oncology
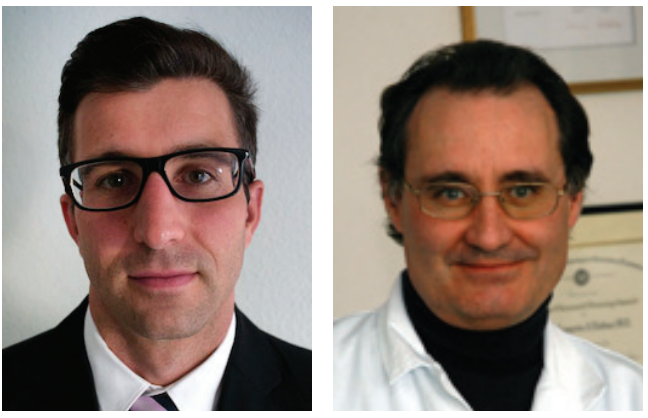

Philippe Kolly ${ }^{1,2}$ \& Jean-François Dufour ${ }^{* 1,2}$
Various therapies are available for hepatocellular carcinoma (HCC) from surgical procedures to systemic therapies. Radiofrequency ablation (RFA) and transarterial embolization are two established therapies. Using electromagnetic energy deposition, the aim of RFA is to induce thermal injury to the tissue [1]. This technique has shown to achieve complete necrosis in $>90 \%$ of the patients with a tumor size of $\leq 3 \mathrm{~cm}[2,3]$ and is therefore considered as a curative option. However, complete necrosis tends to diminish beyond the $3.0 \mathrm{~cm}$ limit, with rates of $71 \%$ for $3.1-5.0 \mathrm{~cm}$ tumors and $25 \%$ for $>5 \mathrm{~cm} \mathrm{[4].} \mathrm{Transcatheter} \mathrm{arterial} \mathrm{emboliza-}$ tion (TAE) is another much investigated therapy for HCC. The goal of this procedure is to achieve an ischemia by performing an intra-arterial embolization of the blood vessel with an embolic agent [1]. TAE can be combined with the local delivery of a chemotherapy such as doxorubicin (TACE). But the main effect of this procedure is due to the embolization $[5,6]$. TAE does rarely achieve a total tumor necrosis [7] and is therefore considered as a palliative option. Nevertheless, this therapy has shown to increase the overall survival (OS) of patients with nonresectable HCC compared with conservative management [8]. Since RFA is limited to early HCC (because of the size limitation) and TAE does not achieve total tumor necrosis, neither RFA nor TAE alone can act as a curative therapy for intermediate HCC.

The American Association for the Study of Liver Diseases (AASLD) and the European Association for the Study of the Liver (EASL) have endorsed the Barcelona Clinic Liver Cancer (BCLC) staging system and treatment algorithm [9,10], which tags patients to various therapies depending on tumor's stage. Based on this scheme [11], RFA is suggested for very early tumors $(\leq 2 \mathrm{~cm})$ that are not candidate for liver transplantation (BCLC 0) and some early tumors (no more than 3 nodules $\leq 3 \mathrm{~cm}$ ) with associated disease (BCLC A). Patients

\section{KEYWORDS}

- ablation - chemoembolization

- HCC • RFA - TACE

'Hepatology, Department of Clinical Research, University of Bern, Bern, Switzerland 
"While combining TACE to RFA for small tumors $(\leq 3 \mathrm{~cm})$ does not improve outcomes, evidences suggest that patients with bigger tumors could benefit from this combination.” with an intermediate tumor burden (BCLC B) are tagged to the palliative TACE therapy. Treatment for this latter stage should probably be more differentiated because of the wide heterogeneity of patients and tumor burden in this stage [12]. Moreover, it has been argued that some patients in that stage may benefit from other treatments that could be curative instead of palliative. One of these suggestions is to combine TAE with RFA in selected cases. Two hypotheses are underlying this idea: first, applying RFA after a round of TAE should give better results than RFA alone and could therefore extend the scope of RFA. The assumption behind this idea is appealing: by reducing the blood flow to the tumor with TAE, there should be less heat loss by convection thus making RFA more effective [13]. The second hypothesis is that the TAE+RFA combination should give better results for intermediate tumors than TAE alone.

Several studies, which mainly differ in the inclusion criterion regarding the size of the tumor, explored the benefit of the TACE-RFA combination against RFA alone. An RCT conducted between 2003 and 2007 with 89 consecutive Japanese patients compared an RFA $(n=43)$ and a TACE-RFA $(n=46)$ group [14]. Inclusion criterion for tumor burden was three or fewer HCC nodules of $\leq 3 \mathrm{~cm}$. Results show that the included patients in both groups mostly had a small single nodule (mean size: $1.7 \mathrm{~cm} \pm 0.5$ ). There was no statistical difference in the overall survival ( $\mathrm{p}$ $=0.515)$ and in the recurrence-free survival $(\mathrm{p}=$ $0.365)$. A meta-analysis of studies investigating the TACE-RFA combination versus RFA only in small HCC $(\leq 3 \mathrm{~cm})$ found no survival benefit for the combination treatment [15]. Those results suggest that the TACE-RFA combination does not improve OS or recurrence-free survival for small HCC. As Wang et al. point out [15], this result is not surprising, knowing that complete necrosis is already achieved in more than $90 \%$ of the small HCC $(\leq 3 \mathrm{~cm})$ treated by RFA $[2,3]$. Therefore, there is probably no need to add another treatment to an already successful therapy.

Two studies extended their inclusion criteria regarding the tumor burden to single HCC of $3.1-5.0 \mathrm{~cm}$. An RCT performed in 2010 with 37 Japanese patients compared TACE-RFA (n = 19) with RFA $(n=18)[16]$. The TACE-RFA group showed better technical success (1.1 RFA sessions vs $1.4 ; \mathrm{p}<0.01)$ and a lower rate of local tumor progression after 3 years of followup ( 6 vs $39 \%$; $\mathrm{p}=0.012$ ). The ablated area was also bigger in the TACE-RFA group ( $\mathrm{p}=0.012)$. According to the authors, this leads to a more spherical ablated area, which could be more effective for local tumor control. Finally, the 3-year OS was not statistically different between the two groups $(\mathrm{p}=0.369)$. Another RCT was conducted in 2011 with 123 Korean patients, comparing TACE-RFA ( $\mathrm{n}=57)$ and RFA ( $\mathrm{n}$ = 66) [17]. TACE-RFA group had a lower local tumor progression rate ( 40 vs $76 \%$; $p<0.001$ ). These two studies suggest that applying TACE before RFA could extend the scope of RFA to the $3.1-5.0 \mathrm{~cm}$ single HCC.

The inclusion criterion was extended beyond the $5 \mathrm{~cm}$ limit by two studies. Yang et al. conducted an RCT in 2008 with tumor ranged from 5.2 to $10.1 \mathrm{~cm}$ in 78 Chinese patients, randomized in four different treatment groups [18]. The TACE-RFA group $(n=24)$ showed $60.3 \%$ of tumor necrosis rate versus $47.8 \%$ for the RFA group $(n=12)$. Tumor relapse rate was lower in the combination group (29.0 vs $34.7 \%$ ). This study provides interesting results but is statistically limited and has a small sample size in the subgroups. Another RCT was conducted in 2013 with 189 Chinese patients with single HCC of $<7 \mathrm{~cm}$ or multiple (up to three) each being $\leq 3$ $\mathrm{cm}$ [19]. They compared TACE-RFA $(\mathrm{n}=94)$ and RFA $(n=95)$. Patients in the TACE-RFA group showed better overall survival $(\mathrm{HR}=0.525, \mathrm{CI}=$ $0.335-0.822 ; \mathrm{p}=0.002)$ and better recurrencefree survival $(\mathrm{HR}=0.575, \mathrm{CI}=0.374-0.897 ; \mathrm{p}$ $=0.009)$. They concluded that TACE-RFA has a better efficiency than RFA for $\mathrm{HCC} \leq 7 \mathrm{~cm}$. This study suffers from an important limitation: while inclusion criterion regarding the size was up to 7 $\mathrm{cm}$, there is no guarantee that TACE-RFA really has better outcome for tumor between 5 and $7 \mathrm{~cm}$ (publishing overall and recurrence-free survival results stratified by tumor size would have been of most interest).

While combining TACE to RFA for small tumors $(\leq 3 \mathrm{~cm})$ does not improve outcomes, evidences suggest that patients with bigger tumors could benefit from this combination. However, all these studies were monocentric and have a small sample size. Evidences are also needed to show that the RFA-TACE combination is better than the recommended TACE procedure. To achieve that, one prospective study conducted by Yin et al. in 2014 [20] with 211 BCLC B classified (single tumor: $5-8 \mathrm{~cm}$ or $2-5$ nodules with diameter $<5 \mathrm{~cm}$ ) Chinese HCC patients. They compared a TACE group $(\mathrm{n}=156)$ and 
a TACE-RFA group $(n=55)$. Patients in the TACE-RFA group had better total tumor necrosis (76.9 vs $46.5 \%$; $\mathrm{p}<0.001)$, a better tumor control rate after 6 months (74.5 vs $54.5 \%$; p $<0.001)$ and a better post-treatment OS (1-, 3 -, 5-year $89.8,61.1$, and $37.4 \%$ vs $67.2,36.6$, and $16.5 \% ; \mathrm{p}=0.01$ ). Despite the interesting result of this study, it potentially suffers from important selection bias: only patients who were considered as potential candidates underwent the RFA procedure. The RCT conducted by Yang et al. [18] also had a TACE arm $(\mathrm{n}=11)$. The TACE-RFA group achieved better tumor necrosis $(60.3 \%)$ than the TACE group $(37.5 \%)$ and a lower tumor relapse rate (29 vs $45.8 \%$ ).

The TAE-RFA combination needs stronger evidence. Especially in that setting, the use of TAE instead of TACE should be privileged, since the hypothesis for this association is the reduction of heat loss as a result of the embolization. A multicenter RCT with intermediate HCC patients should be conducted, comparing three groups: RFA only, TAE-RFA and TAE. By finding the characteristics of patients with intermediate HCC who may benefit from a TAE-RFA combination over TAE, more patients could undergo a curative therapy.

Financial \& competing interests disclosure

$J F$ Dufour is on advisory committees for Bayer, BMS, Gilead Science, Janssen Cilag, Jennerex, Merck, Novartis, engages in speaking and teaching for Bayer, BoehringerIngelheim, Novartis and has a research grant from Bayer. The authors have no other relevant affiliations or financial involvement with any organization or entity with a financial interest in or financial conflict with the subject matter or materials discussed in the manuscript apart from those disclosed.

No writing assistance was utilized in the production of this manuscript.

\section{References}

1 Lencioni R. Loco-regional treatment of hepatocellular carcinoma. Hepatology 52, 762-773 (2010).

2 Livraghi T, Goldberg SN, Lazzaroni S, Meloni F, Solbiati L, Gazelle GS. Small hepatocellular carcinoma: treatment with radio-frequency ablation versus ethanol injection. Radiology 210, 655-661 (1999).

3 Shibata T, Shibata T, Maetani Y, Isoda H, Hiraoka M. Radiofrequency ablation for small hepatocellular carcinoma: prospective comparison of internally cooled electrode and expandable electrode 1. Radiology 238, 346-353 (2006).

4 Livraghi T, Goldberg SN, Lazzaroni S et al. Hepatocellular carcinoma: radio-frequency ablation of medium and large lesions 1 . Radiology 214, 761-768 (2000).

5 Cammà $\mathrm{C}$, Schepis $\mathrm{F}$, Orlando $\mathrm{A}$ et al. Transarterial chemoembolization for unresectable hepatocellular carcinoma: meta-analysis of randomized controlled trials. Radiology 224, 47-54 (2002).

6 Marelli L, Stigliano R, Triantos C et al. Transarterial therapy for hepatocellular carcinoma: which technique is more effective? A systematic review of cohort and randomized studies. Cardiovasc. Intervent. Radiol. 30, 6-25 (2007).

7 Lo CM, Ngan H, Tso WK et al. Randomized controlled trial of transarterial lipiodol chemoembolization for unresectable hepatocellular carcinoma. Hepatology 35,

\section{4-1171 (2002).}

8 Llovet JM, Bruix J. Systematic review of randomized trials for unresectable hepatocellular carcinoma: chemoembolization improves survival. Hepatology 37, 429-442 (2003).

9 Bruix J, Sherman M. Management of hepatocellular carcinoma: an update. Hepatology 53, 1020-1022 (2011).

10 European Association for the Study of the Liver. EASL-EORTC clinical practice guidelines: management of hepatocellular carcinoma. J. Hepatol. 56, 908-943 (2012).

11 Bruix J, Gores GJ, Mazzaferro V. Hepatocellular carcinoma: clinical frontiers and perspectives. Gut 63, 844-855 (2014).

12 Bolondi L, Burroughs A, Dufour JF et al. Heterogeneity of patients with intermediate (BCLC B) hepatocellular carcinoma: proposal for a subclassification to facilitate treatment decisions. Semin. Liver Dis. 2012, 348-359 (2012).

13 Rossi S, Garbagnati F, Lencioni R et al. Percutaneous radio-frequency thermal ablation of nonresectable hepatocellular carcinoma after occlusion of tumor blood supply. Radiology 217, 119-126 (2000).

14 Shibata T, Isoda H, Hirokawa Y, Arizono S, Shimada K, Togashi K. Small hepatocellular carcinoma: is radiofrequency ablation combined with transcatheter arterial chemoembolization more effective than radiofrequency ablation alone for treatment? Radiology 252, 905-913 (2009).
15 Wang W, Shi J, Xie WF. Transarterial chemoembolization in combination with percutaneous ablation therapy in unresectable hepatocellular carcinoma: a meta-analysis. Liver Int. 30, 741-749 (2010).

16 Morimoto M, Numata K, Kondou M, Nozaki A, Morita S, Tanaka K. Midterm outcomes in patients with intermediate-sized hepatocellular carcinoma. Cancer 116, 5452-5460 (2010).

17 Kim JH, Won HJ, Shin YM et al. Mediumsized $(3.1-5.0 \mathrm{~cm})$ hepatocellular carcinoma: transarterial chemoembolization plus radiofrequency ablation versus radiofrequency ablation alone. Ann. Surg. Oncol. 18, 1624-1629 (2011).

18 Yang P, Liang M, Zhang Y, Shen B. Clinical application of a combination therapy of lentinan, multi-electrode RFA and TACE in HCC. Adv. Ther. 25, 787-794 (2008).

19 Peng ZW, Zhang YJ, Chen MS et al. Radiofrequency ablation with or without transcatheter arterial chemoembolization in the treatment of hepatocellular carcinoma: a prospective randomized trial. J. Clin. Oncol. 31, 426-432 (2013).

20 Yin X, Zhang L, Wang YH et al. Transcatheter arterial chemoembolization combined with radiofrequency ablation delays tumor progression and prolongs overall survival in patients with intermediate (BCLC B) hepatocellular carcinoma. BMC Cancer 14 , 849 (2014). 